\section{Utilización de un compuesto de ácidos grasos hiperoxigenados en el tratamiento de heridas crónicas superficiales de diferentes etiologías}

\section{Use of a hyperoxygenated fatty acid compound in the treatment of superficial chronic wounds of different etiologies}

\author{
Teresa Segovia Gómez' \\ Mariano Bermejo Martínez² \\ Joan-Enric Torra-Bou ${ }^{3, *}$
}

1. Enfermera. Exresponsable de la Unidad Multidisciplinar de Úlceras por Presión y Heridas Crónicas, Hospital Universitario Puerta de Hierro, Majadahonda, Madrid. Experta en el Cuidado de Heridas Crónicas acreditado por el GNEAUPP. Miembro del Comité Director del GNEAUPP. Profesora del Curso de Experto Universitario en Heridas Crónicas GNEAUPP-Universidad de Castilla la Mancha.

2. Enfermero. Consulta de Cirugía Vascular y Unidad de Pie Diabético, Hospital Universitario Ramón y Cajal, Madrid, España. Experto en Valoración y Tratamiento HC UCLM y GNEAUPP. Miembro del Comité Consultor del GNEAUPP.

3. Enfermero. Doctor en Ciencias de la salud por la Universidad de Alicante. Máster en gestión e investigación en heridas crónicas por la Universidad de Cantabria. Investigador post-doc de la Facultat d'Infermeria i Fisioteràpia de la Universitat de Lleida. Miembro del Comité Director del GNEAUPP. Trustee del European Pressure Ulcer Advisory Panel (EPUAP), Consultant Advisor, Lindsay Leg Club y colaborador del 4DHealth-Innovation Simulation Center. Miembro del Grup de Recerca en Cures de la Salut (GRECS), IRB Lleida, Universitat de Lleida; colaborador del grupo TR2Lab, Universitat de Vic-Universitat Central de Catalunya e Investigador de la Cátedra de Estudios Avanzados en Heridas de la Universidad de Jaén-GNEAUPP-Fundación Sergio Juán Jordán.

${ }^{*}$ Autor para correspondencia.

Correo electrónico: jetorrabou@hotmail.com (Joan-Enric Torra i Bou).

\section{RESUMEN}

Introducción: La atención a las lesiones cutáneas relacionadas con la dependencia, las úlceras de extremidad inferior y otro tipo de heridas superficiales representa un importante reto para los profesionales de la salud. Estos tipos de lesiones, cuando son superficiales, requieren de un abordaje específico por el elevado riesgo de que se conviertan en heridas más profundas, que pueden afectar a la salud y a la calidad de vida de quienes las padecen, y pueden requerir de tratamientos más complejos y costosos. Los ácidos grasos hiperoxigenados (AGHO) están considerados hoy en día como una de las medidas más importantes para la prevención de este tipo de lesiones. La familia Mepentol ${ }^{\circledR}$ (Mepentol, Mepentol Leche y Mepentol AGHO) son los únicos AGHO clasificados como productos sanitarios con Marca CE clase IIb y, por ello, pueden utilizarse también en el tratamiento de lesiones superficiales. La coexistencia de piel íntegra, pero agredida, y lesiones superficiales es una situación muy habitual en la práctica clínica. Pacientes, material y métodos: Se presentan los resultados

de una serie de casos clínicos en los que se ha utilizado Mepentol $^{\oplus}$ y

Mepentol ${ }^{\oplus}$ Leche para el tratamiento de lesiones crónicas superficiales.

Se incluyeron pacientes con heridas crónicas superficiales de diferente etiología que no fuesen alérgicos a ninguno de los componentes de dichos productos y que dieron su consentimiento a participar en la evaluación. Las lesiones fueron incluidas en la evaluación hasta conseguir su cicatrización o resolución. Resultados: La serie de casos clínicos comprende 54 pacientes con 73 lesiones crónicas superficiales: 45 úlceras de la extremidad inferior,

10 lesiones cutáneas relacionadas con la dependencia, 5 lesiones cutáneas relacionadas con la insuficiencia venosa y 13 lesiones de otra tipología. La antigüedad mediana de las lesiones era de 39 días. Cuarenta y cinco lesiones

$(61,6 \%)$ fueron tratadas con Mepentol $^{\oplus}$ y $28(38,4 \%)$ con Mepentol $^{\odot}$ Leche. Las 73 lesiones incluidas en el estudio cicatrizaron en una mediana de 23 días. No se presentó ninguna complicación ni episodio de infección

local. Discusión: La utilización de Mepentol ${ }^{\oplus}$ y Mepentol ${ }^{\circledR}$ Leche ha demostrado ser efectiva en la cicatrización de heridas crónicas superficiales de diferentes etiologías. La utilización de ambos productos en el cuidado

\section{ABSTRACT}

Introduction: The care of dependency-related skin injuries, lower limb ulcers and other types of superficial wounds, represents a major challenge for healthcare professionals. These types of injuries, when superficial, require a specific approach because of the high risk of developing into deeper wounds, which can affect the health and quality of life of sufferers, and may require more complex and costly treatments. Hyperoxygenated fatty acids (HOFA) are nowadays considered as one of the most important measures for the prevention of these types of injuries. The Mepentol ${ }^{\odot}$ range of products (Mepentol ${ }^{\oplus}$, Mepentol ${ }^{\oplus}$ Leche and Mepentol AGHO) are the only HOFA CE certifies medical devices IIb and can therefore also be used in the treatment of superficial lesions. The coexistence of integrated, but damaged skin, and superficial lesions is a very common situation in clinical practice. Patients, material and methods: The results of a clinical case series in which $\mathrm{Mepentol}^{\oplus}$ and $\mathrm{Mepentol}^{\circ}$ Leche have been used for the treatment of superficial chronic wounds are presented. Patients with superficial chronic wounds of different etiology who were not allergic to any of the components of these products and who consented to participate in the evaluation were included. Lesions were included in the evaluation until healing or resolution was achieved. Results: The case series comprised 54 patients with 73 superficial chronic lesions: 45 lower extremity ulcers, 10 skin lesions related to dependence, 5 skin lesions related to venous insufficiency and 13 lesions of other types. The median age of the lesions was 39 days. 45 lesions $(61.6 \%)$ were treated with $\mathrm{Mepentol}^{\circledR}$ and 28 (38.4\%) with Mepentol ${ }^{\oplus}$ Leche. The 73 lesions included in the study healed in a median of 23 days. There were no complications or episodes of local infection. Discussion: The use of $\mathrm{Mepentol}^{\oplus}$ and $\mathrm{Mepentol}^{\oplus}$ Leche has been shown to be effective in the healing of superficial chronic wounds of different etiologies. The use of both products 
de la piel en pacientes a riesgo, que frecuentemente se asocia a lesiones superficiales, es una excelente opción para mejorar los resultados de prevención y tratar precoz y efectivamente dichas lesiones superficiales.

PALABRAS CLAVE: Ácidos grasos hiperoxigenados, tratamiento, heridas superficiales, heridas crónicas, úlceras de extremidad inferior, lesiones cutáneas relacionadas con la dependencia, insuficiencia venosa. in skin care in patients at risk, which is often associated with superficial lesions, is an excellent option to improve prevention outcomes and to treat these superficial lesions early and effectively.

KEYWORDS: Hyperoxygenated fatty acids, treatment, superficial wounds, chronic wounds, lower extremity ulcers, dependency-related skin lesions, venous insufficiency.

\section{У INTRODUCCIÓN}

La atención a las lesiones cutáneas relacionadas con la dependencia $(\mathrm{LCRD})^{1}$, entre las que podemos destacar lesiones por presión (LPP) o por presión en combinación con cizalla, lesiones cutáneas asociadas a la humedad (LESCAH) ${ }^{2}$, lesiones por fricción, desgarros cutáneos (en inglés "skin tears") y lesiones mixtas o combinadas, constituyen hoy en día un importante problema de salud que requiere diferentes abordajes terapéuticos según la profundidad de las lesiones (tabla 1).

Según datos del $5^{\circ}$ Estudio Nacional de lesiones por presión y otras lesiones cutáneas relacionadas con la dependencia en Espańa, con datos de pacientes adultos y pediátricos de hospitales ${ }^{4,5}$, pacientes sociosanita$\operatorname{rios}^{6}$ y pacientes de atención primaria ${ }^{7}$, las LCRD son un problema de alta prevalencia en todos los niveles asistenciales y la mayoría de ellas son superficiales (tabla 2).

En lo que hace referencia a las úlceras de extremidad inferior (UEI), estas, de acuerdo con el Consenso Nacional de Úlceras de la Extremidad Inferior (CONUEI), también pueden clasificarse en cuatro categorías en cuanto a su profundidad: I en el caso de la lesión que afecta a epidermis y dermis, II si afecta al tejido celular subcutáneo o hipodermis, III la que afecta a la fascia y al músculo, y IV la que afecta al hueso ${ }^{8}$.

La prevalencia de las UEI en España es de 0,89 casos de úlceras venosas por cada mil habitantes, 0,12 casos de úlceras arteriales y 0,27 episodios de úlcera de pie diabético; además, la prevalencia de las úlceras mixtas es de 0,26 por cada mil habitantes, siendo en su mayor parte de etiología venosa. Dicha prevalencia guarda una relación directa con la edad, multiplicándose por dos cuando se considera una población de más de 65 años $^{9}$. No existen datos epidemiológicos acerca de la profundidad de las lesiones en pacientes con UEI.

Tanto en el caso de las LCRD como en las UEI tenemos que añadir, a sus datos de prevalencia, varios elementos que caracterizan a estas lesiones, como la cronicidad, la tasa de recurrencia y la duración de los tratamientos, entre otros, que afectan significativamente a la calidad de vida de pacientes y familiares. Por esta razón, un objetivo fundamental en el cuidado avanzado de heridas debe ser prevenir dichas lesiones, y en el caso de que aparezcan, tratarlas cuanto antes y de manera eficaz para preservar la salud y la calidad de vida de quienes las sufren, intentando reducir sus importantes repercusiones físicas, sociales, psicológicas, y también el alto impacto económico, tanto a nivel individual como para el Sistema de Salud, que estas representan, en dimensiones como la utilización de recursos humanos, materiales e institucionales ${ }^{10-12}$.

Hoy en día, el "gold standard" en el tratamiento local de una herida crónica o de larga duración es la utilización de apósitos basados en la cura en ambiente húmedo $(\mathrm{CAH})^{13}$, los cuales crean en el lecho de la herida unas condiciones favorables a su cicatrización. De todas maneras, este tipo de tratamiento está indicado en lesiones con un determinado nivel de extensión y profundidad, y tanto en el caso de las LCRD como en el de las UEI con frecuencia nos encontramos con situaciones en las que coexisten la piel íntegra, pero agredida, junto a pequeńas lesiones con piel dańada; en estos casos es importante combinar dos acciones

Tabla 1. Clasificación-categorización de las lesiones cutáneas relacionadas con la dependencia

\begin{tabular}{|c|c|c|c|}
\hline Lesiones por presión & $\begin{array}{l}\text { Lesiones combinadas } \\
\text { o multicausales }\end{array}$ & Lesiones por fricción & Lesiones por humedad \\
\hline $\begin{array}{l}\text { Categoría I } \\
\text { Eritema que no palidece en piel } \\
\text { intacta }\end{array}$ & $\begin{array}{l}\text { Categoría I } \\
\text { Eritema que no palidece en piel } \\
\text { intacta }\end{array}$ & $\begin{array}{c}\text { Categoría I } \\
\text { Eritema sin flictena }\end{array}$ & $\begin{array}{l}\text { Categoría 1a } \\
\text { Eritema leve moderado sin pérdida } \\
\text { de integridad cutánea }\end{array}$ \\
\hline $\begin{array}{c}\text { Categoría II } \\
\text { Lesión en epidermis, dermis }\end{array}$ & $\begin{array}{c}\text { Categoría II } \\
\text { Lesión en epidermis, dermis }\end{array}$ & $\begin{array}{c}\text { Categoría II } \\
\text { Lesión con flictena }\end{array}$ & $\begin{array}{l}\text { Categoría 1b } \\
\text { Eritema intenso sin pérdida } \\
\text { de integridad cutánea }\end{array}$ \\
\hline $\begin{array}{c}\text { Categoría III } \\
\text { Lesión en epidermis, dermis y tejido } \\
\text { celular subcutáneo }\end{array}$ & $\begin{array}{l}\text { Categoría III } \\
\text { Lesión en epidermis, dermis y tejido } \\
\text { celular subcutáneo }\end{array}$ & $\begin{array}{c}\text { Categoría III } \\
\text { Lesión con pérdida de integridad } \\
\text { cutánea }\end{array}$ & $\begin{array}{c}\text { Categoría } 2 a \\
\text { Eritema con pérdida de integridad } \\
\text { cutánea (<50\% de la superficie } \\
\text { del eritema) }\end{array}$ \\
\hline $\begin{array}{c}\text { Categoría IV } \\
\text { Lesión hasta estructura articular, } \\
\text { hueso o cartílago }\end{array}$ & $\begin{array}{c}\text { Categoría IV } \\
\text { Lesión hasta estructura articular, } \\
\text { hueso o cartílago }\end{array}$ & & $\begin{array}{c}\text { Categoría } 2 \text { b } \\
\text { Eritema con pérdida de integridad } \\
\text { cutánea (>50\% de la superficie } \\
\text { del eritema) }\end{array}$ \\
\hline
\end{tabular}


Tabla 2. Prevalencia y severidad de los diferentes tipos de lesiones cutáneas relacionadas con la dependencia en España (Estudio Nacional GNEAUPP 2017)

\begin{tabular}{|c|c|c|c|c|}
\hline Tipo de lesión & Hospitales (adultos) & Hospitales (pediatría) & Centros sociosanitarios & $\begin{array}{l}\text { Atención primaria } \\
\text { (pacientes en atención } \\
\text { domiciliaria) }\end{array}$ \\
\hline Todas las LCRD & Prevalencia: $8,7 \%$ & Prevalencia: $4,6 \%$ & Prevalencia: $6,2 \%$ & Prevalencia: $6,1 \%$ \\
\hline LPP & Prevalencia: $7 \%$ & Prevalencia: $3,3 \%$ & Prevalencia: 4,03\% & Prevalencia: 4,8\% \\
\hline LPP Categoría I & $29,9 \%$ & $44,4 \%$ & $14,8 \%$ & $11,1 \%$ \\
\hline LPP Categoría II & $34,9 \%$ & $41,7 \%$ & $34,8 \%$ & $41,3 \%$ \\
\hline LPP Categoría III & $20 \%$ & $11,1 \%$ & $20,6 \%$ & $13,3 \%$ \\
\hline LPP Categoría IV & $11 \%$ & $2,8 \%$ & $18,1 \%$ & $11,4 \%$ \\
\hline Lesión tejidos profundos (LTP) & $1,1 \%$ & - & $1,9 \%$ & $1 \%$ \\
\hline No clasificable & $1,6 \%$ & - & $1,6 \%$ & $0,6 \%$ \\
\hline No conocida o informada & - & - & - & $9,2 \%$ \\
\hline LESCAH & Prevalencia: $1,4 \%$ & Prevalencia: $1,6 \%$ & Prevalencia: $2,2 \%$ & Prevalencia: $1,4 \%$ \\
\hline Categoría la & $42,8 \%$ & $28,6 \%$ & $33,8 \%$ & $36,4 \%$ \\
\hline Categoría lb & $18,7 \%$ & $7,1 \%$ & $29,4 \%$ & $19,7 \%$ \\
\hline Categoría lla & $30,1 \%$ & & $22,1 \%$ & $21,2 \%$ \\
\hline Categoría llb & $5,4 \%$ & & $2,9 \%$ & $3 \%$ \\
\hline No conocida o informada & - & $64,3 \%$ & $11,8 \%$ & $19,7 \%$ \\
\hline Lesiones por fricción & Prevalencia: $0,9 \%$ & - & Prevalencia: $0,8 \%$ & Prevalencia: 1,81 \\
\hline Categoría I & $41,5 \%$ & Sin datos & $11,1 \%$ & $32,3 \%$ \\
\hline Categoría II & $40,7 \%$ & Sin datos & $63 \%$ & $37,1 \%$ \\
\hline Categoría III & $13,6 \%$ & Sin datos & $18,5 \%$ & $12,9 \%$ \\
\hline Categoría IV & - & Sin datos & - & - \\
\hline No conocida o informada & - & Sin datos & $7,4 \%$ & $17,8 \% \%$ \\
\hline ERLADESC & Prevalencia: $0,9 \%$ & Prevalencia: $0,1 \%$ & Prevalencia: $1,31 \%$ & Prevalencia: $1,05 \%$ \\
\hline Superficial & Sin datos & Sin datos & $87,2 \%$ & $92 \%$ \\
\hline No conocida o informada & Sin datos & Sin datos & $12,8 \%$ & $8 \%$ \\
\hline Lesiones combinadas & Prevalencia: $1,5 \%$ & Prevalencia: $0,5 \%$ & Prevalencia: $1 \%$ & Prevalencia: $1 \%$ \\
\hline Categoría I & $16,8 \%$ & Sin datos & $4,9 \%$ & $14,1 \%$ \\
\hline Categoría II & $54,2 \%$ & Sin datos & $51,2 \%$ & $50 \%$ \\
\hline Categoría |II & $2,1 \%$ & Sin datos & $12,2 \%$ & $1,6 \%$ \\
\hline Categoría IV & $23,2 \%$ & Sin datos & $2,4 \%$ & $14,1 \%$ \\
\hline Lesión tejidos profundos (LTP) & $0,5 \%$ & Sin datos & $4,9 \%$ & $1,6 \%$ \\
\hline Lesiones no clasificables & $0,5 \%$ & Sin datos & $2,4 \%$ & - \\
\hline No conocida o informada & - & Sin datos & $21,9 \%$ & $18,8 \%$ \\
\hline Lesiones no clasificadas & Prevalencia: $0,4 \%$ & Sin datos & Prevalencia: $0,35 \%$ & Prevalencia: 0,24\% \\
\hline
\end{tabular}

ERLADESC: erosiones, laceraciones, desgarros cutáneos; LCRD: lesiones relacionadas con la dependencia; LESCAH: lesiones cutáneas asociadas a la humedad; LPP: lesiones por presión. Fuente: GNEAUPP47

que muchas veces no se pueden conseguir con apósitos de CAH debido a que estos, bajo la óptica del coste/efectividad, se utilizan en lesiones más severas, y a su vez no permiten actuar de manera simultánea en el cuidado continuo una o dos veces al día de la piel sana y la reversión de problemas relacionados con la anoxia y el estrés exudativo en la matriz extracelular ${ }^{14}$.

Es aquí donde desempeñan un papel muy importante los ácidos grasos hiperoxigenados (AGHO), un tipo de producto que nos va a permitir un óptimo cuidado de la piel y la reversión de los mencionados procesos anóxicos en los procesos de reperfusión ${ }^{15}$, los cuales se empezaron a desarrollar y utilizar a partir de las evidencias acerca del efecto de los ácidos grasos esenciales ${ }^{16}$.
Existen en el mercado diferentes compuestos de AGHO con diferencias en cuanto a su composición y proceso de fabricación, lo que define perfiles diferentes de acción que en ningún momento pueden extrapolarse de un producto a otro. Mepentol $^{\oplus}$ y Mepentol $^{\oplus}$ Leche (Alfasigma) tienen numerosas evidencias científicas experimentales ${ }^{17-25}$ así como una extensa experiencia clínica ${ }^{25-35}$ que avala su uso en el mantenimiento de la piel en condiciones fisiológicas óptimas: mejoran la microcirculación sanguínea, de forma rápida y prolongada, evitando la isquemia del tejido cutáneo; favorecen la renovación de las células epidérmicas, mejorando la resistencia de la piel; y aportan un grado óptimo de hidratación, evitando la sequedad cutánea y restaurando el film hidrolipídico. Estas actividades proporcionan una excelente prevención primaria, mejorando el estado de la piel para que no aparezcan futuras lesiones. 
A lo largo de los años, el amplio uso de los AGHO de la gama Mepentol $^{\circledR}$ en la práctica clínica ha llevado a enfermería a ampliar sus usos y a aplicarlos directamente en pequeńas heridas donde está afectada la epidermis y dermis superficial; una tipología de paciente muy habitual en los diferentes ámbitos asistenciales, donde coexisten zonas enrojecidas y zonas donde se ha dañado la integridad cutánea. A tal efecto, se vio que dichas heridas cicatrizaban y, asimismo, proporcionan también una excelente prevención secundaria, es decir, que aplicando estas medidas tratamos al paciente de manera precoz, ayudando a tener menos secuelas y que el abordaje general de la lesión sea más fácil. Esta nueva aplicación de los AGHO se sustentaba en los trabajos de Cardoso et al. ${ }^{36,37}$.

Es justamente en este punto donde radica la necesidad de desarrollar una investigación más profunda de la actividad cicatrizante de los AGHO para confirmar la práctica clínica y valorar la eficacia de esta posible actividad. A nivel de estudios preclínicos, se llevaron a cabo varias evaluaciones en torno a la capacidad cicatrizante. Los resultados demuestran que tanto Mepentol $^{\oplus}$ como $^{\text {Mepentol }}{ }^{\oplus}$ Leche favorecen el proceso de cicatrización de heridas, induciendo la migración de células endoteliales y la síntesis de colágeno I y III ${ }^{38,39}$.

Si lo analizamos con detalle, la migración de células endoteliales es un paso esencial para la regeneración de vasos sanguíneos y reparación de heridas. En condiciones estacionarias, las células muestran un perfecto entramado de microfilamentos, que mantienen a las células en contacto con su sustrato de adhesión; en cambio, cuando cambian a un estado migratorio, estos microfilamentos desaparecen, permitiendo así que estas células migren. En este sentido, Mepentol $^{\oplus}$ y Mepentol $^{\circledR}$ Leche han demostrado inducir tanto la migración como la proliferación celular ${ }^{38,39}$.

Igualmente, en la cicatrización están implicados numerosos procesos, entre los que destacan aquellos que conducen a la degradación del tejido dañado y los que permiten disponer de nuevas moléculas de la matriz extracelular específicas, siendo los colágenos los más importantes. Una adecuada reposición de las proteínas de la matriz degradadas en el proceso de reconstrucción tisular es uno de los procesos esenciales de una cicatrización exitosa. Mepentol $^{\circledR}$ y Mepentol $^{\circledR}$ Leche demostraron inducir la síntesis de colágeno I y III de forma dependiente de la dosis ${ }^{38,39}$.

Otro aspecto que hay que abordar es la categorización de los productos. De acuerdo con la legislación europea, Mepentol $^{\oplus}$ y Mepentol ${ }^{\oplus}$ Leche son productos sanitarios con Marca CE clase IIb, únicos en el mercado español.

Dentro de los productos sanitarios existen diferentes categorías. Así, estos se pueden clasificar en diferentes clases que condicionan su utilización y las regulaciones que dichos productos deben cumplir: I, IIa, IIb y III. La adscripción de cada producto a una clase determinada se lleva a cabo de acuerdo con los criterios establecidos para cada uno de ellos ${ }^{40}$.

En la clase I se clasifican los productos destinados a ser utilizados en la piel como barrera, para la compresión o para la prevención, actuando siempre en piel intacta. Sin embargo, dentro de la clase II nos encontramos como clase IIa aquellos productos destinados a actuar en el microentorno de la herida, y en la clase IIb, aquellos productos destinados principalmente a ser utilizados con heridas que hayan producido una ruptura de la dermis.

Esta clasificación en clase II, y a diferencia de la clase I, requiere de estudios preclínicos y clínicos que demuestren su eficacia y garanticen su uso; en el caso de la clase IIb, en heridas abiertas.

\section{У PACIENTES, MATERIAL Y MÉTODO}

Con el objetivo de evaluar la efectividad de la utilización de Mepentol $^{\circledR}$ (Alfasigma), un compuesto de AGHO en forma de aceite, y Mepentol ${ }^{\circledR}$
Leche (Alfasigma), en forma de emulsión en la cicatrización de heridas superficiales, se realizó una serie de casos clínicos en pacientes atendidos en la consulta de la unidad multidisciplinar de heridas del Hospital Universitario Puerta de Hierro de Majadahonda o bien en las unidades de hospitalización por miembros de la mencionada unidad (ver casos 1 a 7).

Mepentol $^{\circledast}$ es un producto sanitario, indicado para la prevención y tratamiento de LPP en estadio I y II, que se compone principalmente de AGHO junto con dos extractos de plantas, Equisetum arvense e Hypericum perforatum. E. arvense (cola de caballo) tiene como propiedades el ser elastificante, reepitelizante, reafirmante y tonificante, y $H$. perforatum (hierba de San Juan) actúa como lenitivo, antiséptico, dermoprotector y reepitelizante.

Mepentol ${ }^{\oplus}$ Leche es igualmente un producto sanitario, indicado para la prevención y tratamiento de las UEI en estadios iniciales, que se compone principalmente de $\mathrm{AGHO}$ junto con dos extractos de plantas, Aloe vera y Mimosa tenuiflora. A. vera tiene propiedades lenitivas, hidratantes y estimulantes del crecimiento epitelial y $M$. tenuiflora actúa como cicatrizante e hidratante.

Su mecanismo de acción se basa en ${ }^{38,39,41-44}$ :

- Mejorar la microcirculación sanguínea, evitando la isquemia del tejido cutáneo.

- Favorecer la renovación de las células epidérmicas, mejorando la resistencia de la piel y facilitando la cicatrización.

- Restaurar el film hidrolipídico, evitando la deshidratación cutánea.

- Crear una barrera antimicrobiana, que protege la zona ulcerada de agentes externos.

- Evitar el dańo causado por un aumento de los radicales libres, gracias a su actividad antioxidante.

El ácido graso mayoritario en $\mathrm{Mepentol}^{\oplus}$ y Mepentol $^{\oplus}$ Leche es el ácido oleico ( $\omega-9)$.

Se conoce que los ácidos grasos (AG) tienen un papel importante y una implicación terapéutica potencial en la curación de heridas cutáneas. Existen evidencias de que los AG linolénico ( $\omega$-3), linoleico ( $\omega-6)$ y oleico $(\omega-9)$ pueden modular el cierre de heridas de la piel, pero se ha observado que concretamente el ácido oleico $(\omega-9)$ induce más rápidamente el cierre de las heridas en comparación con las otras dos familias de AG en un estudio experimental in vivo sobre modelo de ratones al que se les induce una herida por abrasión ${ }^{36,37}$.

Los extractos de plantas en ambas formulaciones actúan reforzando el componente activo principal y responsable de la eficacia terapéutica, que son los AGHO. La prueba de ello se confirma con la publicación del estudio clínico de Candela Zamora et al. ${ }^{29}$, estudio comparativo entre Mepentol $^{\oplus}$ y Mepentol ${ }^{\bullet} \mathrm{AGHO}$, este último producto formulado con la misma base de AGHO, pero sin los extractos de plantas presentes en Mepentol $^{\circ}$ y de venta exclusivamente hospitalaria, cuyos resultados evidencian que en ambos grupos de tratamiento conseguimos revertir las UPP de categoría I, confirmando que se trata de productos equivalentes.

Se incluyeron en la evaluación pacientes con heridas superficiales [lesiones por presión de categoría II; lesiones por dermatitis asociada a la incontinencia (DAI) de categoría $\mathrm{II}^{3}$; úlceras de extremidad inferior de grado I, tales como úlceras venosas y úlceras de pie diabético ${ }^{8}$; lesiones cutáneas relacionadas con la insuficiencia venosa, así como otras heridas superficiales de diferentes etiologías en la extremidad inferior]. Se excluyó de la evaluación a pacientes con lesiones profundas o que declinasen participar por cualquier motivo en la evaluación. Asimismo, fueron excluidos pacientes con alergia a algunos de los componentes de Mepentol $^{\oplus}$ o Mepentol ${ }^{\circledR}$ Leche. Las lesiones fueron incluidas en el estudio hasta conseguir su total cicatrización o resolución. 
Se recogió información demográfica de los pacientes, así como datos relativos a las lesiones (tipo, localización, síntomas relacionados con el cuidado de la piel, antigüedad, tipo de AGHO utilizado, pauta de tratamiento y tiempo hasta la resolución).

Se consideró como variable de respuesta el tiempo necesario para la cicatrización o resolución de las lesiones. Se elaboró una base de datos en Excel con la información relativa a los pacientes y a sus lesiones, y esta fue analizada mediante estadísticos descriptivos con el paquete estadístico IBM SPSS Statistics versión 25.

Los pacientes fueron informados de los objetivos de la evaluación y firmaron un consentimiento informado para participar en la evaluación y permitir la realización de fotografías para evaluar la evolución de las lesiones. El equipo de investigación garantizó en todo momento el anonimato de la información relativa a los pacientes. El estudio no representó ningún coste añadido ni para los pacientes ni para la institución, ya que se utilizaron los productos objeto de evaluación en un contexto de práctica clínica habitual de acuerdo con el protocolo del centro.

La recogida de información se desarrolló entre los ańos 2009 y 2014.

\section{$\checkmark$ RESULTADOS}

Durante el período de realización de la evaluación se incluyeron en el estudio 54 pacientes con un total de 73 lesiones. La edad media de los 54 pacientes era de 73,3 9 (DE) años (intervalo de confianza [IC] al $95 \%$ : 70,6;75,5). Veintisiete de los pacientes eran mujeres $(50 \%)$ y 27 (50), hombres.

En la tabla 3 se presenta información acerca de las variables demográficas de los pacientes, así como la distribución por tipo de lesiones.

Un $57,4 \%$ de los pacientes presentaban insuficiencia venosa, un 1,9\% insuficiencia arterial, un 50\% diabetes y un 29,6\% obesidad. Se calculó el índice tobillo/brazo (ITB) en 27 de los pacientes con úlceras venosas con un valor medio de 1,05 $\pm 0,14$ (DE) (IC95\%: 0,99;1,1).

En la tabla 4 se resume la información acerca de la sintomatología relacionada con el cuidado de la piel.

La antigüedad mediana de las lesiones era de 39 días (mínimo: 5 días y máximo: 270) (IC 95\% de la media: 40; 61,6 días).

Cuarenta y cinco lesiones (un 61,6\%) fueron tratadas con Mepentol ${ }^{\circ}$ y $28(38,4 \%)$, con Mepentol Leche. Las 73 lesiones incluidas en el estudio cicatrizaron en una mediana de 23 días (mínimo: 4 días y máximo: 124 días) (IC 95\% de la media: 21,8; 32,6 días). En la tabla 5 se describe el número de aplicaciones diarias según tipo de AGHO.

En el transcurso de la realización de la evaluación, ningún paciente abandonó el tratamiento ni se produjo ninguna reacción adversa o problema relacionado con los productos objeto de evaluación; tampoco ningún episodio de infección local.

En 42 lesiones se utilizó como apósito secundario un apósito de gasa; en 27, un apósito de gasa junto a un vendaje de malla; en 2, una espuma polimérica sin borde, y en otros 2 una espuma con adhesivo de silicona. En 26 lesiones se combinó el tratamiento de AGHO con otras medidas terapéuticas: en 19 casos con medias de compresión, en 3 con calcetines de compresión, en 2 con un fieltro de descarga y en 2 con talonera.

Por lo que respecta a las 73 lesiones superficiales tratadas, su tipología era la siguiente: 45 UEI (43 úlceras venosas y 2 lesiones de pie diabético), 10 LCRD, 5 lesiones cutáneas por insuficiencia venosa y 13 otros tipos de lesiones (5 lesiones traumáticas, 4 lesiones dermatológicas, 2 lesiones infecciosas, 1 lesión por rascado y 1 lesión por picadura).

Han cicatrizado la totalidad de las lesiones tratadas con Mepentol $^{\varpi}$, 45 lesiones, y con Mepentol $^{\circledR}$ Leche, 28 lesiones.

En la tabla 6 se describen las lesiones y su evolución según tipo de lesión.

Tabla 3. Información de los pacientes incluidos en el estudio

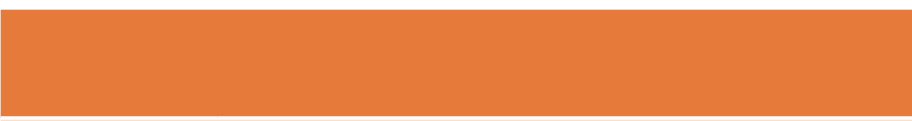

Edad

Número de lesiones por paciente

\section{Úlceras de la extremidad inferior (UEI)}

Tipo de Lesiones cutáneas relacionadas con la dependencia (LCRD) lesiones Lesiones cutáneas relacionadas con la insuficiencia venosa Otro tipo de lesiones

\begin{tabular}{|c|c|c|}
\hline $\begin{array}{l}\text { Todos } \\
\text { N: } 54\end{array}$ & $\begin{array}{l}\text { Mujeres } \\
\text { N: } 27(50 \%)\end{array}$ & $\begin{array}{l}\text { Hombres } \\
\text { N: } 27(50 \%)\end{array}$ \\
\hline $\begin{array}{l}73,3 \pm 9 \text { (DE) años } \\
\text { (IC95\%: 70,6;75,5) }\end{array}$ & $\begin{array}{l}71,8 \pm 9,5 \text { (DE) años } \\
\text { (IC } 95 \%: 68,1 ; 75,6)\end{array}$ & $\begin{array}{l}74,2 \pm 8,5 \text { (DE) años } \\
\text { (IC } 95 \%: 70,1 ; 77,6)\end{array}$ \\
\hline \multicolumn{3}{|c|}{$p: 0,23$} \\
\hline $\begin{array}{c}1,26 \pm 0,58 \text { (DE) lesiones } \\
\text { (IC 95\%: } 1,1 ; 1,4)\end{array}$ & $\begin{array}{l}1,18 \pm 0,62 \text { (DE) lesiones } \\
\text { (IC 95\%: 0,93;1,43) }\end{array}$ & $\begin{array}{l}1,33 \pm 0,55 \text { (DE) lesiones } \\
\text { (IC 95\%: 1,1,1;1,52) }\end{array}$ \\
\hline $45(61,6 \%)$ & $24(53,3 \%)$ & $21(46,7 \%)$ \\
\hline $10(13,7 \%)$ & $6(60 \%)$ & $4(40 \%)$ \\
\hline $5(6,8 \%)$ & $1(20 \%)$ & $4(80 \%)$ \\
\hline $13(17,2 \%)$ & $6(46,2 \%)$ & $7(53,8 \%)$ \\
\hline
\end{tabular}

Tabla 5. Pautas de aplicación diaria de los ácidos grasos hiperoxigenados

Tabla 4. Síntomas relacionados con el cuidado de la piel

\begin{tabular}{l}
\hline Eritema \\
\hline Dolor \\
\hline Edema \\
\hline Prurito \\
\hline Escozor \\
\hline Descamación y sequedad \\
\hline
\end{tabular}

\begin{tabular}{|r|}
$88,9 \%$ \\
$85,2 \%$ \\
$63 \%$ \\
$61,1 \%$ \\
$53,7 \%$ \\
$9,3 \%$ \\
\hline
\end{tabular}

\begin{tabular}{|l|c|r|}
\hline & Mepentol $^{\circledR}$ & Mepentol $^{\circledR}$ Leche \\
\hline 1 vez cada 2 días & $2(4,4 \%)$ & \\
\hline 2 veces al día & $10(22,2 \%)$ & $2(7,1 \%)$ \\
\hline 3 veces al día & $33(73,3 \%)$ & $26(92,1 \%)$ \\
\hline
\end{tabular}


Tabla 6. Descripción y evolución de las lesiones

\begin{tabular}{|c|c|c|c|c|}
\hline & $\begin{array}{l}\text { Úlceras de extremidad } \\
\text { inferior (UEI) (N: 45) }\end{array}$ & $\begin{array}{l}\text { Lesiones cutáneas relacionadas } \\
\text { con la dependencia (N: 10) }\end{array}$ & $\begin{array}{l}\text { Lesiones cutáneas } \\
\text { relacionadas con la } \\
\text { insuficiencia venosa (N: } 5 \text { ) }\end{array}$ & $\begin{array}{c}\text { Otros tipos } \\
\text { de lesiones (N: 13) }\end{array}$ \\
\hline Localización & $\begin{array}{l}\text { Tercio inferior de la pierna, } 32(71,1 \%) \\
\text { Tercio medio de la pierna, } 5(11,1 \%) \\
\text { Toda la pierna, } 5(11,1 \%) \\
\text { Pie, } 3(6,6 \%)\end{array}$ & $\begin{array}{l}\text { Sacro, } 4(40 \%) \\
\text { Glúteo, } 2(20 \%) \\
\text { Talón, } 2(20 \%) \\
\text { Tercio medio pierna, } 1(10 \%) \\
\text { Dedo pie, } 1(10 \%)\end{array}$ & $\begin{array}{l}\text { Tercio medio pierna, } 2 \text { (40\%) } \\
\text { Toda la pierna, } 3(60 \%)\end{array}$ & $\begin{array}{l}\text { Toda la pierna, } 2(15,4 \%) \\
\text { Tercio medio pierna, } 2(15,4 \%) \\
\text { Tercio inferior pierna, } 4(30,8 \%) \\
\text { Pie, } 5(38,5 \%)\end{array}$ \\
\hline Antigüedad & $\begin{array}{l}\text { Mediana: } 42 \text { días } \\
\text { Valor mínimo: } 16 \\
\text { Valor máximo: } 270\end{array}$ & $\begin{array}{l}\text { Mediana: } 8,5 \text { días } \\
\text { Valor mínimo: } 5 \\
\text { Valor máximo: } 33\end{array}$ & $\begin{array}{l}\text { Mediana: } 49 \text { días } \\
\text { Valor mínimo: } 29 \\
\text { Valor máximo: } 68\end{array}$ & $\begin{array}{l}\text { Mediana: } 35 \text { días } \\
\text { Valor mínimo: } 14 \\
\text { Valor máximo: } 98\end{array}$ \\
\hline $\begin{array}{l}\text { Problemas } \\
\text { asociados }\end{array}$ & $\begin{array}{l}\text { Dolor, } 41(91,1 \%) \\
\text { Eritema, } 39(86 \%) \\
\text { Prurito, } 33(86,7 \%) \\
\text { Edema, } 23(51,1 \%) \\
\text { Escozor, } 20(44,4 \%) \\
\text { Descamación y sequedad, } 4(8,9 \%)\end{array}$ & $\begin{array}{l}\text { Eritema, } 10(100 \%) \\
\text { Dolor, } 7(70 \%) \\
\text { Prurito, } 4(40 \%) \\
\text { Edema, } 4(40 \%) \\
\text { Escozor, } 3(30 \%) \\
\text { Descamación y sequedad, } 2(20 \%)\end{array}$ & $\begin{array}{l}\text { Edema, } 5(100 \%) \\
\text { Descamación y sequedad, } 5(100 \%) \\
\text { Eritema, } 4(80 \%) \\
\text { Prurito, } 4(80 \%) \\
\text { Escozor, } 3(60 \%) \\
\text { Dolor, } 1(20 \%)\end{array}$ & $\begin{array}{l}\text { Dolor, } 12(92 \%) \\
\text { Edema, } 12(92 \%) \\
\text { Eritema, } 11(85 \%) \\
\text { Escozor, } 9(69 \%) \\
\text { Prurito, } 6(46 \%) \\
\text { Descamación y sequedad, } 2(15,4 \%)\end{array}$ \\
\hline Tipo de AGHO & $\begin{array}{l}\text { Aceite, } 31(68,9 \%) \\
\text { Emulsión, } 14(31,1 \%)\end{array}$ & $\begin{array}{l}\text { Aceite, } 9(90 \%) \\
\text { Emulsión, } 1(10 \%)\end{array}$ & $\begin{array}{l}\text { Aceite, } 2(40 \%) \\
\text { Emulsión, } 3(60 \%)\end{array}$ & $\begin{array}{l}\text { Aceite, } 3(23,1 \%) \\
\text { Emulsión, } 10(76,9 \%)\end{array}$ \\
\hline $\begin{array}{l}\text { Pauta de } \\
\text { tratamiento }\end{array}$ & $\begin{array}{l}10(22,2 \%) \text { dos veces al día } \\
35(77,8 \%) \text { tres veces al día }\end{array}$ & $\begin{array}{l}2(20 \%) \text { cada } 2 \text { días } \\
2(20 \%) \text { dos veces al día } \\
6(60 \%) \text { tres veces al día }\end{array}$ & $5(100 \%)$ tres veces al día & $13(100 \%)$ tres veces al día \\
\hline $\begin{array}{l}\text { Tiempo para } \\
\text { resolución }\end{array}$ & $\begin{array}{l}\text { Mediana } 26 \text { días } \\
\text { Mínimo: } 7 \text { días } \\
\text { Máximo: } 124 \text { días }\end{array}$ & $\begin{array}{l}\text { Mediana } 5,5 \text { días } \\
\text { Mínimo: } 4 \text { días } \\
\text { Máximo: } 29 \text { días }\end{array}$ & $\begin{array}{l}\text { Mediana } 28 \text { días } \\
\text { Mínimo: } 12 \text { días } \\
\text { Máximo: } 33 \text { días }\end{array}$ & $\begin{array}{l}\text { Mediana } 15 \text { días } \\
\text { Mínimo: } 7 \text { días } \\
\text { Máximo: } 23 \text { días }\end{array}$ \\
\hline
\end{tabular}

AGHO: ácidos grasos hiperoxigenados; UEl: úlcera de extremidad inferior

\section{У DISCUSIÓN}

La muestra de lesiones incluidas en la serie de casos es representativa de las lesiones superficiales relacionadas con las LCRD y las UEI y es un exponente de la práctica asistencial habitual del cuidado de heridas, es decir evidencia de la práctica clínica real.

La totalidad de las lesiones han cicatrizado en el transcurso de la evaluación clínica. Las lesiones incluidas en la serie presentaban una mediana de 39 días de evolución, y para su cicatrización precisaron una mediana de 23 días, un poco más de la mitad de su tiempo de evolución.

En ninguna de las lesiones en tratamiento han aparecido nuevas lesiones superficiales ni episodios de infección local; además, se ha conseguido mejorar todos aquellos síntomas y signos relacionados con dichas lesiones, tales como prurito, dolor, eritema, descamación, sequedad y edema. Estos síntomas ocasionan grandes molestias a los pacientes y constituyen un riesgo de aparición de nuevas lesiones o de agravamiento de las existentes.

En ningún caso se han presentado efectos adversos asociados al uso de Mepentol ${ }^{\oplus}$ y Mepentol $^{\oplus}$ Leche. La utilización de Mepentol ${ }^{\oplus}$ y Mepentol ${ }^{\circledR}$ Leche ha sido totalmente compatible con otras medidas para el tratamiento etiológico.

La estrategia de tratamiento utilizada ha sido totalmente aceptada por los pacientes y ninguno de ellos ha manifestado malestar en relación con la misma, expresando una gran parte de los pacientes una mejora o resolución de los síntomas asociados a su patología cutánea.
El cuidado de la piel en zonas de alto riesgo como las lesiones incluidas en el estudio requiere de una valoración constante para la detección y el tratamiento precoz de posibles lesiones incidentes. La utilización de AGHO tanto en la prevención como en el tratamiento de lesiones incipientes y superficiales ha demostrado ser una medida terapéutica eficaz frente a otros abordajes como la utilización de apósitos de $\mathrm{CAH}$ que impiden el cuidado local frecuente de la piel y la detección precoz de lesiones.

La utilización de Mepentol $^{\oplus}$ y Mepentol $^{\oplus}$ Leche ha demostrado en nuestra serie de casos ser efectiva en el abordaje de la prevención de nuevas lesiones cutáneas y en el tratamiento de las lesiones superficiales existentes, lo que bajo la óptica de la economía de la salud implica un ahorro importante en el coste relacionado con apósitos, curas y tiempo de enfermería frente al abordaje de este tipo de lesiones con apósitos de CAH.

A tenor de los resultados de nuestra serie de casos clínicos, el primer estudio publicado sobre el uso de AGHO (Mepentol ${ }^{\oplus}$ y Mepentol $^{\oplus}$ Leche) en heridas superficiales en una serie grande de pacientes y a falta estudios con diseños más robustos como ensayos clínicos, que tienen la dificultad ańadida de conseguir grupos homogéneos en cuanto a características basales de paciente y lesiones y su abordaje en entornos de práctica clínica habitual, el presente trabajo aporta un primer nivel de evidencia que sustenta la utilización de productos de AGHO con marcado $\mathrm{CE}$ Clase IIb en el tratamiento de lesiones superficiales. Al respecto es importante destacar la importancia de que estos productos estén certificados como Marca CE clase IIb, lo que avala su seguridad en entornos de lesiones cutáneas con ruptura de la piel. 


\section{$\vee$ CONCLUSIONES}

Los AGHO en aceite o emulsión con marcado CE clase IIb (Mepentol ${ }^{\circ}$ y Mepentol ${ }^{\circ}$ Leche, Alfasigma) son una medida coste-efectiva y segura en el tratamiento de heridas superficiales, que además es totalmente compatible con la prevención de lesiones cutáneas en pacientes con o en riesgo de LCRD y de UEI.

Es importante destacar que en el mercado también existe Mepentol ${ }^{\circ}$ AGHO, igualmente con marca CE clase IIb, versión de Mepentol $^{\circ}$ de venta exclusiva en centros hospitalarios en envase clínico y sin extractos de plantas, con una eficacia equivalente a $\mathrm{Mepentol}^{\circ}$ demostrada en un estudio comparativo ${ }^{29}$.

La utilización de Mepentol $^{\curvearrowleft}$ y Mepentol ${ }^{\triangleright}$ Leche ha demostrado ser una excelente medida de prevención primaria y son los únicos productos de AGHO que pueden ser utilizados en la prevención secundaria (tratamiento precoz) de lesiones superficiales, a tenor de

\section{CASO 1}
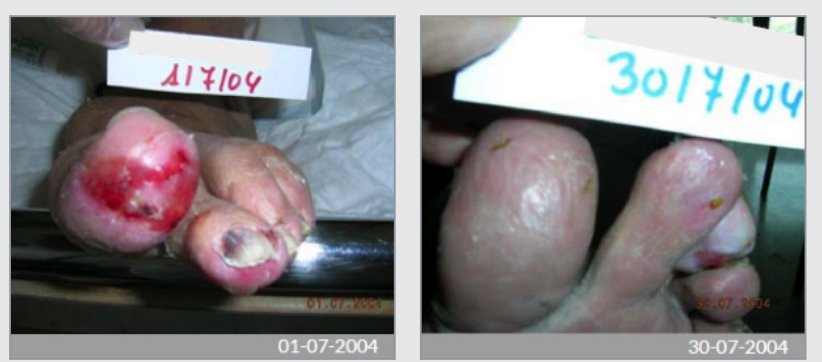

- Antecedentes: varón, 65 años, diabético.

- Exploración: LPP de categoría II en dedo.

- Tratamiento: AGHO aceite, 3 veces al día, y plantilla de descarga.

- Tiempo hasta la cicatrización: 29 días.

\section{CASO 2}
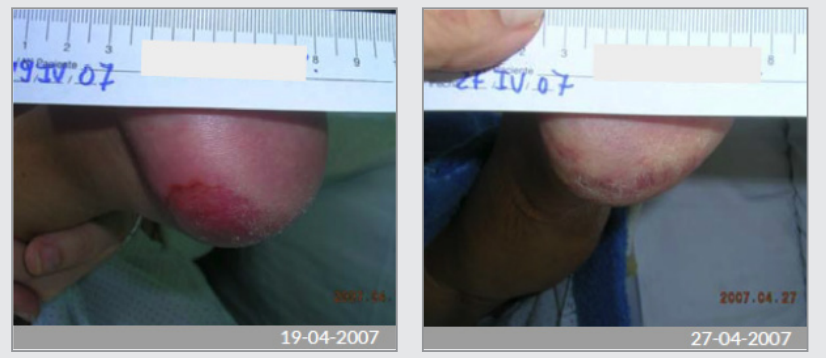

- Antecedentes: mujer, 91 años.

- Exploración: LPP de categoría II en talón con eritema circundante (categoría I).

- Tratamiento: AGHO aceite, 3 veces al día, gasa y talonera para alivio de presión.

- Tiempo hasta la cicatrización: 8 días. nuestros resultados, una excelente relación coste/beneficio que permite disminuir el sufrimiento de los pacientes y ahorrar costes en el tratamiento de lesiones que potencialmente pueden agravarse con el impacto que ello conlleva en tratamientos locales mucho más caros, complicaciones, alargamiento de estancias hospitalarias y mayor tiempo de enfermería

\section{Conflicto de intereses}

Los autores declaran no tener ningún interés comercial en los productos objeto de evaluación. TSG y JETB han sido ponentes en actividades de divulgación de parte de los resultados del presente estudio sin remuneración económica por parte del laboratorio fabricante. Los autores TSG, MBM y JETB han contribuido al diseño de la evaluación. TSG y MBM han recogido los datos. JETB ha realizado el diseńo final del protocolo de evaluación, así como el análisis estadístico de los resultados, y ha dirigido la elaboración del informe de evaluación.

\section{CASO 3}
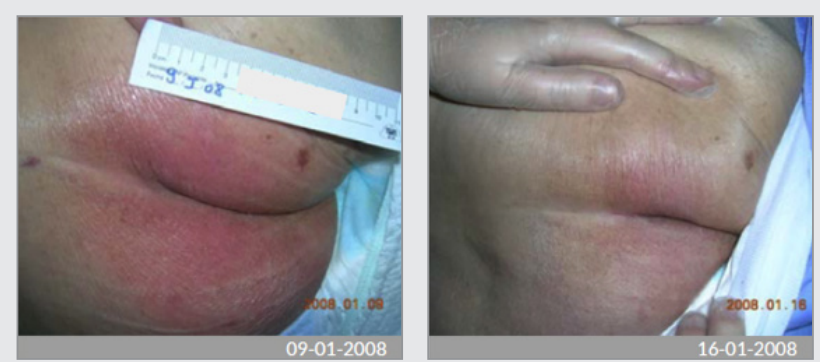

- Antecedentes: mujer, 78 años, incontinencia.

- Exploración: úlcera por humedad Ib en glúteos y pequeńa zona con LPP categoría II.

- Tratamiento: AGHO aceite, 2 veces al día.

- Tiempo hasta la cicatrización: 7 días.

\section{CASO 4}
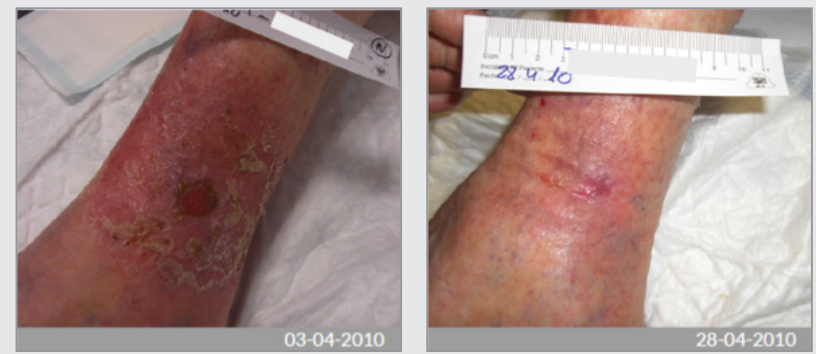

- Antecedentes: mujer, 72 años, diabetes mellitus insulinodependiente, insuficiencia respiratoria e insuficiencia venosa (ITB 1).

- Exploración: lesión de grado II y piel perilesional afectada.

- Tratamiento: AGHO aceite, 3 veces al día, gasa y medias de compresión.

- Tiempo hasta la cicatrización: 25 días. 


\section{CASO 5}
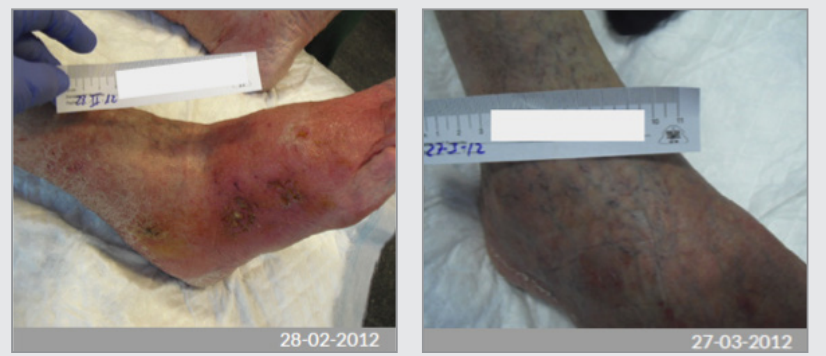

- Antecedentes: varón, 81 años, diabetes mellitus insulinodependiente, hipertensión arterial e insuficiencia respiratoria.

- Exploración: lesiones neuropáticas de grado II en pie derecho, edema y eritema.

- Tratamiento: AGHO aceite, 3 veces al día, gasa y calcetín algodón.

- Tiempo hasta la cicatrización: 28 días.

\section{CASO 6}
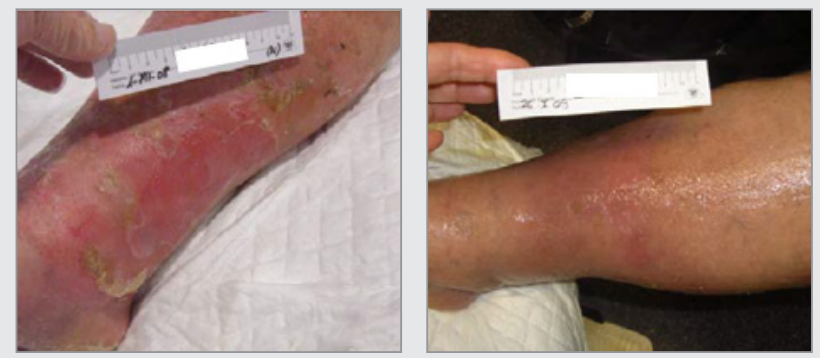

- Antecedentes: varón, 55 años, obesidad mórbida.

- Exploración: lesiones venosas, prurito, escozor intenso y dolor moderado (ITB: 1,1 ).

- Tratamiento: AGHO emulsión, 3 veces al día, compresa gasa y Tubifix.

- Tiempo hasta la cicatrización: 1 mes y 26 días.

\section{CASO 7}
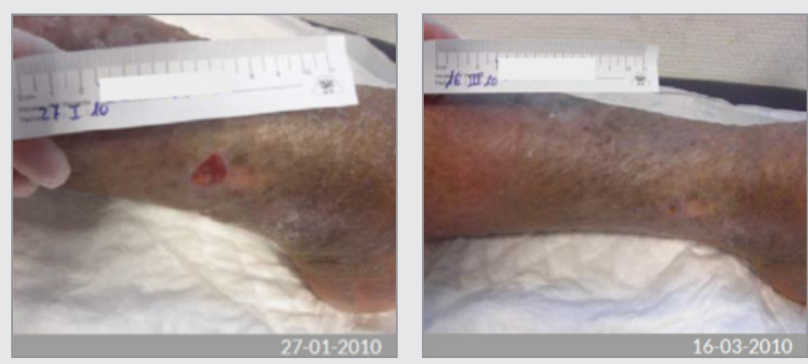

- Antecedentes: varón, 78 años, insuficiencia venosa crónica, infarto agudo miocardio hace 3 años, diabetes mellitus insulinodependiente. Sintrom.

- Exploración: úlceras venosas de grado II en ambas piernas, antigüedad 9 meses; descamación y sequedad de piel perilesional.

- Tratamiento: AGHO emulsión, 3 veces al día, y venda elástica.

- Tiempo hasta la cicatrización: 1 mes y 17 días.

\section{BIBLIOGRAFÍA}

1. García-Fernández FP, Agreda JJ, Verdú J, Pancorbo-Hidalgo PL. A New Theoretical Model for the Development of Pressure UIcers and Other Dependence-Related Lesions. J Nurs Scholarsh. 2014;46(1):28-38. doi: 10.1111/jnu.12051. Epub 2013 Oct 11

2. Torra-Bou JE, Rodríguez Palma M, Soldevilla Agreda JJ, García Fernández FP. Sarabia Lavín R, Zabala Blanco J, et al. Redefinición del concepto del abordaje de las lesiones por humedad. Una propuesta conceptual y metodológica para mejorar el cuidado de las lesiones cutáneas asociadas a la humedad (LESCAH). Gerokomos. 2013;24(2):90-4

3. García-Fernández FP, Soldevilla-Agreda JJ, Pancorbo-Hidalgo $\mathrm{PL}$, Verdú-Soriano J, López-Casanova P, Rodríguez-Palma M. Clasificación-categorización de las lesiones relacionadas con la dependencia. Serie Documentos Técnicos GNEAUPP n ${ }^{0} 11$. Grupo Nacional para el Estudio y Asesoramiento en Úlceras por Presión y Heridas Crónicas. Logroño, 2014.

4. Pancorbo-Hidalgo PL, García-Fernández FP, Pérez-López C, Soldevilla-Agreda JJ. Prevalencia de lesiones por presión y otras lesiones cutáneas relacionadas con la dependencia en población adulta en hospitales españoles: resultados del $5^{\circ}$ Estudio Nacional de 2017. Gerokomos. 2019;30(2):76-86.

5. Pancorbo-Hidalgo PL, Torra-Bou JE, García-Fernández FP, Soldevilla-Ágreda JJ. Prevalence of pressure injuries and other dependence-related skin lesions among pediatric patients in hospitals in Spain. EWMA J. 2018;19(2):29-37.
6. Soldevilla-Agreda JJ, García-Fernández FP, Rodríguez-Palma M, Torra-Bou JE, Pancorbo-Hidalgo PL. Prevalencia de lesiones por presión y otras lesiones cutáneas relacionadas con la dependencia en residencias de mayores y centros sociosanitarios de España en 2017. Gerokomos. 2019:30(4):192-9.

7. García-Fernández FP, Torra-Bou JE, Soldevilla-Ágreda JJ, Pancorbo-Hidalgo PL. Prevalencia de lesiones por presión y otras lesiones cutáneas relacionadas con la dependencia en centros de atención primaria de salud de España en 2017. Gerokomos. 2019;30(3):134-41

8. Marinel.lo Roura J, Verdú Soriano J, coord. Conferencia nacional de consenso sobre las úlceras de la extremidad inferior (C.O.N.U.E.I.). Documento de consenso 2018. 2. ${ }^{\text {a }}$ ed. Madrid: Ergon; 2018.

9. Torra-Bou JE, Soldevilla Agreda JJ, Rueda López J, Verdú Soriano $J$, Roche Rebollo E, Arboix i Perejamo M, et al. 1 1er estudio nacional de prevalencia de úlceras de pierna en España. Estudio Smith\&Nephew 2002-2003. Gerokomos. 2004;4:230-47.

10. Gorecki C, Brown JM, Nelson EA, Briggs M, Schoonhoven L, Dealey $C$, et al. Impact of Pressure Ulcers on Quality of Life in Older Patients: A Systematic Review. J Am Geriatr Soc. 2009;57(7):1175-83.

11. Pieper B, Langemo D, Cuddigan J. Pressure Ulcer Pain: A Systematic Literature Review and National Pressure Ulcer Advisory Panel White Paper. Ostomy Wound Manage. 2009:55(2):16-31.
12. Torra-Bou JE, García-Fernández FP, Pérez-Acevedo G, Sarabia-Lavín R, Paras-Bravo P, Soldevilla-Ágreda JJ, et al. El impacto económico de las lesiones por presión. Revisión bibliográfica integrativa. Gerokomos. 2017;28(1):30-7.

13. Lagana G, Andreson EH. Moisture dressings: The new standard in wound care. JNP. 2010:6:366-70.

14. Kim JH, Yang B, Tedesco A, Lebig EGD, Ruegger PM, Xu K, et al. High Levels of Oxidative Stress and Skin Microbiome are Critical fo Initiation and Development of Chronic Wounds in Diabetic Mice. Sci Rep. 2019;9(1):19318. doi: 10.1038/s41598-019-55644-3

15. Houwing R, Ovregoor M, Kon M, Jansen G, van Asbeck BS, Haalboom JR. Pressure induced skin lesions in pigs: reperfusion injury and the effects of vitamin E. J Wound Care. 2000:9(1):36-40.

16. Declair $\mathrm{V}$. The usefulness of topical application of essential fatty acids (EFA) to prevent pressure ulcers. Ostomy Wound Manage 1997:43(5):48-54.

17. Romanelli M, Tedeschi A, Piaggesi A, Torra-Bou JE. TcPO2 and

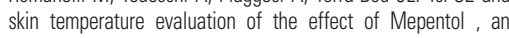
hyper-oxygenated fatty acids solution for the prevention and treatment of stage I pressure ulcers, in the capilar microcirculation before and after heel loading in healthy volunteers. Presentado en el 6th European Pressure Ulcer Advisory Panel Open Meeting - Budapest (Hungary). September, 2002

18. Rueda López J, Segovia Gómez T, Torra-Bou JE, Bermejo Martínez M. Clinical Evaluation of the systematic use of Mepentol ${ }^{\circledR}$ 
an hyper-oxygenated fatty acids solution in the prevention and treatment of stage I pressure ulcers, in an internal medicine nursing ward. Presentado en el 6th European Pressure Ulcer Advisory Panel Open Meeting - Budapest (Hungary). September, 2002.

19. Casaroli R, Reina M, Vilaró S, Torra-Bou JE. "in vitro" evaluation of the effect of Mepento| ${ }^{\circledR}$, a compound of hyperoxigenated fatty acids for the prevention and treatment of stage I pressure ulcers in skin keratynocites improvement. 6th European Pressure Ulcer Advisory Panel Open Meeting - Budapest (Hungary). September, 2002.

20. Rueda-López J, Torra-Bou JE, Segovia-Gómez T, Bermejo-Martíenz M. Laser Doppler assessment of the effect of Mepentol ${ }^{\circledR}$, a compound of hyperoxigenated fatty acids for the prevention and treatment of stage I pressure ulcers, in the capilar microcirculation in the heel of a patient at risk of developing pressure ulcers during a 120 minutes time cycle. 6th European Pressure Ulcer Advisory Panel Open Meeting - Budapest (Hungary). September, 2002.

21. Rueda-López J, Torra-Bou JE, Segovia-Gómez T, Bermejo-Martínez M. Laser doppler blood flow and temperature measurement of Mepentol ${ }^{\circledast}$, a compound of hyperoxigenated fatty acids for the prevention and treatment of stage I pressure ulcers, in the capilar microcirculation in the sacral area of a patient at risk of developing pressure ulcers during a 120 minutes time cycle. 6th European Pressure Ulcer Advisory Panel Open Meeting - Budapest (Hungary). September, 2002.

22. Torra-Bou JE, Segovia-Gómez T, Bermejo-Martínez M. Aplicación tópica de un compuesto de ácidos grasos hiperoxigenados. Efectos preventivos y curativos en úlceras por presión. Determinación experimental en la mejora de la circulación capilar mediante láser doppler. Rev Rol Enf. 2003:26(1):54-61.

23. Polignano R, Torra i Bou JE. Effects of Hyperoxygenated fatty acids in venous leg ulcer microcirculation. Congreso. París, 2004.

24. Sanchez JP, et al. Effectiveness of hyperoxygenated fatty acid in the local oxygenation of patients with diabetic foot. CONGRESO EWMA. European Wound Management Association. Belgium, 2011.

25. Lázaro-Martínez JL, Sánchez-Rios JP, García-Morales E, Cecilia-Matilla A, Segovia-Gómez T. Increased transcutaneous oxygen tension in the skin dorsum over the foot in patients with diabetic foot disease in response to the topical use of an emulsion of hyperoxygenated fatty acids. Int J Lower Wound Extr. 2009;8(4):187-93

26. Torra-Bou JE, Segovia Gómez T, Verdú Soriano BN, Nolasco Bonmatí A, Rueda López J, Arboix i Perejamo M. The effectiveness of a hyperoxygenated fatty acid compound in preventing pressure ulcers. J Wound Care. 2005;14(3):117-21.

27. Gouveia J, Miguéns C, Torra i Bou JE, Gómez T. Ensaio Clínico aberto sobre a efectividade do Mepentol® um composto de ácidos gordos hiperoxigenados, na prevenção de úlceras de pressão nos calcâneos. Nursing. 2006;207:30-5.

28. Díaz Alonso Y, Riveiro Vela AM, González Pérez M. Prevención de úlceras por presión. Resultados de un programa de prevención en unidad de cuidados intensivos pediátricos. Rev ROL Enf. 2009;32(5): 361-6.

29. Candela-Zamora MD, Martín-Gómez MA, Solas-Gómez B, Fernández-Pérez C, Martín-González M, Manzanedo-Basilio L, et al. Estudio comparativo de efectividad de dos ácidos grasos hiperoxigenados en el tratamiento de úlceras de grado I en pacientes geriátricos hospitalizados. Enferm Clin. 2010;20:10-6.

30. Carrasco JM, Dumont E, Ruz A, Gálvez F, Gutiérrez M, Montesinos $P$, et al. La prevención y la constancia conducen a la integridad tisular. Gerokomos. 2011;22(2):1-6.

31. Pérez-Acevedo G, Torra-Bou JE, Manzano Canillas ML, Bosch-Alcaraz A. Management of purpura fulminans skin lesions in a premature neonate with sepsis: a case study. J Wound Care. 2019;28(4):198-203.

32. Puentes Sánchez J, Pardo González CM, Pardo González MB, Navarro Casado FJ, Puentes Sánchez R, Méndez González JM, et al. Prevención de Úlceras vasculares y pie diabético. Rev ROL Enf 2006; 29(10):663-8

33. Segovia Gómez T, Javares Curto T, Barahona M, Verdú Soriano J. Cuidados en piel perilesional o con riesgo de lesión. Resultados de la aplicación de una emulsión de ácidos grasos hiperoxigenados con aloe Barbadensis y Mimosa Tenuiflora. Rev ROL Enf 2007;30(10):43-8.

34. Carrasco Herrero JM, Dumont Lupiañez E, Gálvez Ramírez F, Gutiérrez García M, Montesinos Sánchez P, García González MA. Un antes y un después: del riesgo 0 deterioro de la integridad cutánea a la integridad tisular. Gerokomos. 2008;19(3):153-8.

35. Puentes Sánchez J, Pardo González CM, Pardo González MB Navarro Casado FJ, Puentes Sánchez R, Méndez González JM et al. Evaluación clínica abierta multicéntrica no aleatorizada y prospectiva de la efectividad de una emulsión a base de ácidos grasos hiperoxigenados, Aloe barbadensis y Mimosa tenuiflora (Mepentol ${ }^{\oplus}$ Leche) en diferentes tipos de pacientes con riesgo de ulceración. Gerokomos 2012;23(3):123-7.

36. Cardoso CR, Aparecida Souza M, Vieira Ferro E, Favoreto S, Deolina Oliveira J. Influence of topical administration of $n-3$ and $n-6$ essential and $n-9$ nonessential fatty acids on the healing of cutaneous wounds. Wound Rep Reg. 2004;12:235-43.

37. Cardoso CR, Favoreto S Jr, Oliveira LL, Vancim JO, Barban GB, Ferraz DB. Oleic acid modulation of the immune response in wound healing: a new approach for skin repair. Immunobiology. 2011:216(3):409-15. doi: 10.1016/j.imbio.2010.06.007. Epub 2010 Jul 23. PMID: 20655616.

38. Dossier científico Mepentol y Mepentol AGHO. ALFASIGMA ESPAÑA, S.L. Barcelona s/f.

39. Dossier científico Mepentol Leche. ALFASIGMA ESPAÑA, S.L. Barcelona s/f.

40. Reglamento (UE) 2017/745 del Parlamento Europeo y del Consejo de 5 de abril de 2017 sobre los productos sanitarios, por el que se modifican la Directiva 2001/83/CE, el Reglamento (CE) n. ${ }^{0} 178 / 2002$ y el Reglamento (CE) n. ${ }^{0} 1223 / 2009$ y por el que se derogan las Directivas 90/385/CEE y 93/42/CEE del Consejo. Diario oficial de la Unión Europea. 5.5.2017. Disponible en: https://eur-lex.europa.eu/legal-content/ES/TXT/PDF/?uri=CELEX:32017R0745\&from=ES (acceso 9 de enero de 2020).

41. Castleman M. The Healing Herbs. Emmaus, PA: Rodale Press, 1991. p. 19-221.

42. D'Agostino M. Sterols from Equisetum arvense. Boll Soc Ital Biol Sper 1984:60(12):2241-5.

43. PDR for Herbal Medicines. 2. ed. Montvale: Medical Economics Co., 2000; p. 719-25

44. Villar del Fresno AM, Carretero ME. Fitofarmacia: Revisión Hipérico. Farmacia profesional. 2003;17(5):76-8. 